\title{
Amyloid- $\beta$ and tau pathologies relate to distinctive brain dysconnectomics in preclinical autosomal-dominant Alzheimer's disease
}

Edmarie Guzmán-Vélez ${ }^{12 *}$, Ibai Diez ${ }^{34 *}$, Dorothee Schoemaker ${ }^{1}$, Enmanuelle PardillaDelgado ${ }^{1}$, Clara Vila-Castelar ${ }^{1}$, Joshua T. Fox-Fuller ${ }^{15}$, Ana Baena ${ }^{6}$, Reisa A. Sperling ${ }^{27}$, Keith A. Johnson ${ }^{278}$, Francisco Lopera $^{6}$, Jorge Sepulcre ${ }^{34 * *}, \&$ Yakeel T. Quiroz ${ }^{126 * *}$

${ }^{1}$ Department of Psychiatry, Massachusetts General Hospital, Harvard Medical School, Boston, MA, USA; ${ }^{2}$ Department of Neurology, Massachusetts General Hospital, Harvard Medical School, Boston, MA, USA; ${ }^{3}$ Gordon Center for Medical Imaging, Department of Radiology, Massachusetts General Hospital, Harvard Medical School, Boston, MA, USA; ${ }^{4}$ Athinoula A. Martinos Center for Biomedical Imaging, Department of Radiology, Massachusetts General Hospital, Harvard Medical School, Charlestown, MA, USA; ${ }^{5}$ Department of Psychological and Brain Sciences, Boston University, Boston, MA, USA; ${ }^{6}$ Grupo de Neurociencias, Universidad de Antioquia, Medellín, Antioquia, Colombia; ${ }^{7}$ Center for Alzheimer Research and Treatment, Department of Neurology, Brigham and Women's Hospital, Boston, MA, USA; ${ }^{8}$ Department of Radiology, Massachusetts General Hospital, Harvard Medical School, Boston, MA, USA

* Dr. Guzmán-Vélez and Dr. Diez are co-first authors

${ }^{* *}$ Dr. Quiroz and Dr. Sepulcre are co-senior authors

\section{Corresponding authors:}

Yakeel T. Quiroz, PhD

Associate Professor, Harvard Medical School

Departments of Psychiatry and Neurology, Massachusetts General Hospital $391^{\text {st }}$ Ave, Suite 101, Charlestown, MA 02129 
bioRxiv preprint doi: https://doi.org/10.1101/2021.07.03.450933. this version posted July 4 , 2021. The copyright holder for this preprint (which

was not certified by peer review) is the author/funder, who has granted bioRxiv a license to display the preprint in perpetuity. It is made available under aCC-BY-NC-ND 4.0 International license.

Phone: (617) 643-5944; Fax: (617) 726-5760; E-mail: yquiroz@mgh.harvard.edu

Jorge Sepulcre, MD, PhD

Associate Professor, Harvard Medical School

Gordon Center for Medical Imaging, Massachusetts General Hospital

149 13th St, Suite 5.209, Charlestown, MA 02129

Phone (617) 726-2899; E-mail: jsepulcre-bernad@mgh.harvard.edu

Search terms: familial Alzheimer's disease, fMRI, positron emission tomography, pathology, resting-state functional connectivity, graph theory 


\begin{abstract}
Amyloid- $\beta$ and tau pathology in preclinical Alzheimer's disease (AD) are hypothesized to propagate through brain networks that are critical for neural communication. We used highresolution graph-based network analyses to test whether in vivo amyloid- $\beta$ and tau burden related to segregation and integration of functional brain connections, and their association with memory, in cognitively-unimpaired Presenilin-1 E280A carriers who will develop early-onset AD dementia. Greater tau burden predicted weaker functional segregation and integration of the precuneus with other densely connected regions like the insula and entorhinal cortex, a site of early tau accumulation that is critical for memory. We also observed greater segregation and integration in the striatum and multimodal integrated networks that harbor amyloid- $\beta$ early. Findings enlighten our understanding of how AD-related pathology distinctly alter the brain's functional architecture to interfere with information processing within and across neural systems, possibly contributing to the spread of pathology and ultimately resulting in dementia.
\end{abstract}




\section{INTRODUCTION}

The progressive accumulation of extracellular amyloid- $\beta$ plaques and intracellular neurofibrillary tangles that characterize Alzheimer's disease (AD) begin several years before symptom onset (i.e. the preclinical stage $)^{1,2}$ and follows distinct spatio-temporal patterns ${ }^{3,4}$. Amyloid- $\beta$ accumulates throughout anatomically distant neocortical regions two decades before the onset of memory impairment ${ }^{2,5}$, whereas tau accumulation is first observed in the transentorhinal and entorhinal cortex, later spreading into adjacent association and unimodal cortices ${ }^{6-8}$. These patterns of pathology accumulation in $A D$ are highly reminiscent of functional networks in the brain, which can be represented by the interregional coherence of spontaneous neural activity fluctuations during rest using functional magnetic resonance imaging (fMRI). This has led some to hypothesize that pathology propagation in $A D$ may occur through functional networks ${ }^{9-11}$. In vivo neuroimaging studies have shown that amyloid- $\beta$ first accumulates in a set of distributed brain regions that form part of the so-called default mode network. The DMN includes the precuneus, medial prefrontal and inferior parietal cortices and is considered a backbone of mainly cortical integration and which is strongly associated with episodic memory ${ }^{12,13}$. Conversely, tau pathology begins to accumulate in vulnerable loci (e.g., the transentorhinal and entorhinal cortex), later advancing to local functionally connected regions, possibly following trans-synaptic spread along functional connections ${ }^{8,9,14}$. As such, characterizing how AD-related pathology relates to the brain's functional architecture in the preclinical stage of $A D$, before individuals develop dementia, can improve our understanding of the impact of pathology on neural communication and propagation early in the disease, and help with early detection and prediction of cognitive decline, as well as disease staging.

Some functional brain networks have been shown to undergo two distinct phases that are differentially associated with $A D$ stages. For instance, studies have reported increased functional 
connectivity (i.e., hyperconnectivity) within the DMN in cognitively-unimpaired older adults with high levels of amyloid- $\beta$ but low tau, and reduced functional connectivity (i.e. hypoconnectivity) in those with high levels of both amyloid- $\beta$ and tau ${ }^{15-17}$. Some DMN regions, particularly the precuneus, have also been shown to act as mediators between hyperconnected and hypoconnected hubs ${ }^{16}$, which are brain regions that are highly interconnected and that act as way stations to integrate information across different and often segregated or distributed neural systems. This is perhaps a reflection of changes in neural activity or metabolism associated with neurodegeneration and disease progression. Many of these hubs, including those from the DMN, are particularly vulnerable to early amyloid- $\beta$ pathology accumulation ${ }^{18}$, thus impacting connections that contribute to multiple functional and behavioral processes.

While some studies have suggested an inverse relationship between tau deposition and functional connectivity, particularly in the medial temporal cortex ${ }^{16,19}$, others have shown that elevated tau deposition is associated with stronger local functional connectivity in the early stages of the disease ${ }^{10,20,21}$. Greater functional connectivity in tau pathology hotspots in the preclinical stage of $A D$ have been related to a faster accumulation of tau in interconnected regions, suggesting that functional connectivity in regions that accumulate tau faster and earlier may enable the spread of tau to local or nearby structures that are closely functionally connected ${ }^{10,20,22}$. This is consistent with animal studies demonstrating that neuron-to-neuron propagation of tau pathology is facilitated by greater synaptic connectivity and neuronal activity ${ }^{23-25}$.

These complex brain networks have a modular organization - i.e., different voxels of the brain are organized in communities that specialize on distinct tasks. These modules have a large number of links that connect voxels within a community (network segregation) and a smaller set of links that integrate information between communities (network integration). However, very little is known about how early AD-related pathology-particularly tau pathology burden, a strong 
predictor of neurodegeneration and cognitive decline ${ }^{26-28}$ - distinctly impact the integration and segregation of networks crucial for efficient information processing. Most of the published research has been limited by focusing on amyloid- $\beta$ and not tau, and by studying older adults who are at high risk but may not develop AD-dementia ${ }^{29}$. Further, many studies have examined functional connectivity by averaging sets of pair-wise correlations, thereby obscuring the intricate dynamics of functional networks and potential effects on cognitive functioning in the preclinical stage of $A D$.

To address these shortcomings in the field, we used high-resolution (voxel-level) graph-based network analyses to characterize the topological organization of functional connections in the brain associated with in vivo amyloid- $\beta$ and tau burden, and episodic memory in individuals with autosomal-dominant AD (ADAD). Studying ADAD provides a unique opportunity to understand the associations between $A D$-related pathology and functional connections in preclinical $A D$, as mutation carriers are virtually guaranteed to develop dementia and have very limited age-related comorbidities that could potentially contribute to brain dysfunction. We studied cognitivelyunimpaired individuals from the world's largest kindred with ADAD caused by the E280A mutation in the Presenilin-1 (PSEN1) gene. Mutation carriers have a well-characterized clinical profile and disease trajectory, with an onset of mild cognitive impairment $(\mathrm{MCl})$ at a median age of 44 years $(95 \% \mathrm{Cl}, 43-45)$ and dementia at 49 years $(95 \% \mathrm{Cl}, 49-50)^{30}$, which allows us to estimate how far they are from the onset of cognitive impairment and dementia. Individuals carrying the PSEN1 E280A mutation exhibit widespread cortical amyloid- $\beta$ accumulation approximately two decades before estimated $\mathrm{MCl}$ onset, and tau pathology in the entorhinal cortex close to six years before estimated $\mathrm{MCl}$ onset ${ }^{2,28}$. Similarly, mutation carriers show reduced glucose metabolism in temporal and parietal regions, including in the precuneus more than a decade before estimated $\mathrm{MCl}$ onset ${ }^{31}$. 
In particular, we aimed to elucidate how weighted-degree centrality patterns, known to be important for understanding network organization and information transmission hubs, relate to brain pathology and episodic memory in mutation carriers. We computed a data-driven voxellevel whole-brain analysis to create maps of functional hubs that help characterize segregated (within-network) and integrated (between-network) connections and examined whether the segregation and integration of functional networks at resting differed between mutation carriers and age-matched non-carrier family members, and their relationship to AD-related pathology burden and episodic memory. We hypothesized that there would be significant group differences in the segregation and integration of functional connections in DMN regions, particularly in the precuneus and medial temporal regions as these are the most vulnerable to tau burden in ADAD. We also hypothesized that weaker segregated functional connectivity would be associated with greater tau burden and worse episodic memory, and stronger integrated functional connectivity with higher amyloid- $\beta$ burden and worse episodic memory.

\section{RESULTS}

Sample characteristics. We examined 21 cognitively-unimpaired PSEN1 E280A carriers and 31 non-carriers. Participants performed within normal limits on neuropsychological tests and had a Clinical Diagnostic Rating scale (CDR) score of 0, a Functional Assessment Staging Test (FAST) score of 2 or less, and a Mini-Mental State Examination (MMSE) score of 26 or greater, which denote that they had no objective cognitive impairment. Groups did not significantly differ in age, education, sex, or MMSE scores (Table 1). Compared to non-carriers, mutation carriers performed significantly worse than non-carriers in the Consortium to Establish a Registry for Alzheimer's Disease (CERAD) word list total recall, a measure of episodic memory that is sensitive to early cognitive changes in this population $(F(1,49)=7.87, p=.007, d=0.73)($ Table 1). 


\section{Segregated and integrated functional connectivity patterns differ between cognitively-} unimpaired mutation carriers and non-carriers. We first examined whether there were distinct patterns of segregated and integrated functional connectivity between groups. Higher values in segregated functional connectivity maps denote that a given voxel has a high number of strongly functionally connected links to other regions of the same functional network, whereas higher values in integrated functional connectivity maps mean that the voxel is an important hub for integrating information between brain communities. Compared to non-carriers, mutation carriers showed less segregated functional connectivity in the precuneus, and stronger segregated functional connectivity in the striatum, orbitofrontal cortex, thalamus, and middle and superior frontal gyri (Figure 1). They also showed less segregated functional connectivity in the lateral parietal cortex, inferior temporal gyrus and entorhinal cortex, as well as greater functional connectivity in the anterior cingulate cortex, but none of the latter regions survived correction for multiple comparisons.

Mutation carriers exhibited less integrated functional connectivity in the precuneus, as well as in the posterior hippocampus, medial occipital lobe and brainstem, and greater integrated functional connectivity in the striatum, superior frontal gyrus, paracentral lobule and sensorimotor cortex, compared to non-carriers (Figure 1). There was also less integrated functional connectivity in the lateral temporal cortex and greater integration in the dorsolateral prefrontal cortex; however, none of the latter regions survived correction for multiple comparisons.

\section{Amyloid- $\beta$ and tau burden are distinctively associated with functional connectivity maps}

in cognitively-unimpaired mutation carriers. We then examined changes in functional networks associated with AD progression in the preclinical stage by testing the influence of amyloid- $\beta$ plaques and tau pathology burden, measured with 11C Pittsburg compound B and 
[F18] Flortaucipir PET respectively, on the functional connectivity of segregated and integrated networks in mutation carriers. First, we compared tau pathology burden between mutation carriers and non-carriers and found that, as previously reported ${ }^{2,28}$, the precuneus and entorhinal cortex had the greatest accumulation of tau pathology compared to non-carriers, and the inferior temporal gyrus to a lesser extent (Figure 2A). We then tested how tau burden in these regions predicted segregated and integrated functional connectivity in mutation carriers. We found that greater tau pathology burden predicted lower segregated functional connectivity in the precuneus, angular gyrus, entorhinal cortex, hippocampus, frontal operculum, inferior temporal gyrus, and brainstem (Figure 2B). Greater tau pathology also predicted higher segregated functional connectivity in the medial prefrontal and anterior cingulate cortices, inferior frontal gyrus, medial occipital lobe and the sensorimotor cortex (Figure 2B). In addition, higher tau pathology burden significantly predicted lower integrated functional connectivity in the precuneus, hippocampus and posterior occipital cortex, and greater integrated functional connectivity in the anterior medial occipital lobe, the right sensorimotor cortex, and the paracentral lobule (Figure 2B).

We then identified the largest clusters of voxels (>100 voxels) and examined their relationship with pathology burden in five regions of interest that have the highest accumulation of amyloid- $\beta$ and tau pathology in the preclinical stage as shown by our data and prior research ${ }^{2,28,32}$, including amyloid- $\beta$ in the striatum and precuneus, and tau in the entorhinal cortex, precuneus and inferior temporal gyrus (Figure 2A). Specifically, in PSEN1 E280A carriers there is elevated $\mathrm{PiB}$ binding in the striatum and precuneus approximately two decades before estimated onset of $\mathrm{MCl}^{32-34}$, followed by tau in the entorhinal cortex and precuneus, and later inferior temporal gyrus approximately six years before the onset of $\mathrm{MCl}$. As such, while these data are crosssectional, examining the relationship of functional connectivity with each of these regions could provide insight into how functional connections may change as the disease progresses. We 
found that higher segregated functional connectivity in the inferior frontal gyrus and sensorimotor cortices were most strongly related to higher tau in the entorhinal cortex, while higher segregated functional connectivity in the anterior cingulate cortex and medial occipital lobe was most strongly associated with higher tau burden in the inferior temporal cortex (Figure 2B). Together, these findings suggest that segregated functional connectivity in these regions increase when there is greater tau burden compared to amyloid- $\beta$. Further, higher segregated functional connectivity in the frontal operculum and entorhinal cortex was most strongly associated with less tau burden similarly in all three regions, while higher segregated functional connectivity in the precuneus was most strongly related to less tau in the inferior temporal cortex. In turn, segregated functional connectivity in the hippocampus was associated with amyloid- $\beta$ and tau pathology in all brain regions to a similar degree. As such, these data suggest that segregated functional connectivity on the frontal operculum, precuneus and entorhinal cortex decrease in the presence of greater tau accumulation compared to amyloid- $\beta$ burden.

In regard to the integration of functional connections, we found that stronger functional connectivity in the sensorimotor cortex was most strongly associated with greater tau burden in the entorhinal cortex, stronger functional connectivity in the medial occipital lobe and superior frontal gyrus with higher tau burden in the inferior temporal gyrus, and stronger functional connectivity in the paracentral lobule with greater tau burden in the precuneus (Figure 2B). Greater integration of functional connections in these regions were overall associated with less amyloid- $\beta$ in the striatum and precuneus. Conversely, weaker integrated functional connectivity in the precuneus was related to higher tau burden most strongly compared to amyloid- $\beta$ burden (Figure 2B). Again, these data raise the possibility that integrated functional connectivity weakens or strengthens in these regions as the distinct pathologies accumulate in the five apriori selected regions. 


\section{Functional connectivity alterations are associated with worse episodic memory in}

cognitively-unimpaired mutation carriers. Finally, we examined whether functional maps predicted episodic memory performance in mutation carriers, as measured by the word list delayed recall test. We found that worse total recall scores were significantly associated with higher segregated functional connectivity in subcortical regions including the entorhinal cortex, hippocampus, amygdala, insula and basal ganglia, and with lower functional connectivity in the medial prefrontal cortex and right supramarginal gyrus (Figure 3). Associations between worse delayed recall scores and higher segregated functional connectivity were observed in the medial occipital cortex and inferior temporal gyrus, and with lower segregated functional connectivity in the anterior cingulate, ventrolateral prefrontal, sensorimotor and superior temporal cortices, but did not survive multiple comparisons. Worse delayed recall scores were also significantly associated with greater integrated functional connectivity in subcortical regions that include the thalamus, amygdala, hippocampus and insula, and the brainstem, as well as lower integrated functional connectivity in the medial occipital lobe, somatosensory cortex and paracentral lobule. Relationships between worse delayed recall scores and higher integrated functional connectivity in the medial prefrontal cortex, precuneus, posterior cingulate cortex and inferior temporal gyrus, and lower integrated functional connectivity in the superior frontal gyrus and posterior temporal lobe, did not survive multiple comparisons.

\section{DISCUSSION}

The human brain is a complex system composed of neuronal networks that are critical for the efficient processing of information across brain structures. These complex brain networks have a modular topology, where brain regions are organized into communities that form internally dense and externally sparse subnetworks that specialize on distinct tasks and higher-order 
cognitive functions. It has been proposed that AD-related pathology spreads along neuronal functional connections, disrupting neural activity and communication that eventually results in cognitive dysfunction and dementia. As such, understanding how the brain's functional architecture is impacted by pathology in the preclinical stage of $A D$, before individuals exhibit cognitive impairment, may significantly assist with predicting disease progression and risk for dementia, as well as with the development of novel therapeutics. In this study, we used PET imaging, resting-state fMRI, and high-resolution graph theory techniques to test the association of in vivo amyloid- $\beta$ and tau pathology with patterns of segregation and integration of functional networks, and their relationship with episodic memory, in a unique ADAD cohort with a homogeneous clinical profile and predictable age of $\mathrm{MCl}$ and dementia onset.

We provide novel in vivo evidence that amyloid- $\beta$ and tau pathology predict distinct patterns of segregation and integration of functional networks that resemble the patterns of pathology accumulation in AD and relate to episodic memory in preclinical ADAD. As hypothesized, we found that the precuneus, one of the earliest sites of pathology accumulation in ADAD, exhibited significantly weaker functional segregation and integration of networks compared to agematched non-carriers. This decrease in the functional segregation and integration of the precuneus with other regions was also strongly associated with greater tau burden in the entorhinal cortex, precuneus and inferior temporal gyrus. We observed that compared to amyloid- $\beta$ pathology in the striatum and precuneus, segregated functional connectivity in particular appeared to be significantly reduced in the presence of tau pathology, suggesting that functional dysconnectivity between the precuneus and local regions is most evident as carriers get closer to the age of $\mathrm{MCl}$ onset. The precuneus is a major association area that is considered to be essential for integrating internally- and externally-driven information, and for supporting complex cognition and behavior such as episodic memory, visuospatial processing and selfrelated processing (e.g., empathy, perspective taking) ${ }^{35-37}$. The precuneus also has projections 
to cortical and subcortical structures including adjacent areas of the posteromedial cortex, lateral parietal cortex, dorsolateral prefrontal cortex and somatomotor regions, heteromodal association areas, cingulate cortex, thalamus, striatum and brainstem ${ }^{38}$. Functionally, the precuneus is a core hub of the DMN with dense connections to many structures across the brain. As such, the dysconnectivity seemingly induced by tau pathology in the precuneus both within and across brain regions may significantly contribute to the behavioral and cognitive changes observed as mutation carriers approach $\mathrm{MCl}$ onset.

Notably, the entorhinal cortex also exhibited decreased segregated functional connectivity associated with greater tau pathology, but not changes in network integration, suggesting that only connections to locally or intrinsically connected regions to entorhinal cortex are weakened in the presence of tau pathology. Prior studies have shown that the entorhinal cortex and precuneus fire synchronously and have high metabolic activity during resting, which in turn has been associated with greater vulnerability to tau deposition ${ }^{25,39,40}$. Specifically, strong functional connections in older individuals with elevated amyloid- $\beta$ levels have been shown to increase the probability of tau seeding and spreading in $A D^{10}$, which may help explain why tau is first seen in the entorhinal cortex and precuneus in PSEN1 E280A carriers. This elevated functional connectivity (inverted-U pattern) in the earlier stages of AD likely switches to a decrease in functional connectivity in the presence of tau pathology and possibly neurodegeneration that is evident closer to $\mathrm{MCl}$ onset. In fact, prior studies have shown that individuals at high risk for $\mathrm{AD}$ exhibit hyperconnectivity, particularly in the DMN, before a reduction in functional connectivity can be observed ${ }^{15}$. Specifically, the hyperconnectivity phase has been most strongly associated with amyloid- $\beta$ pathology and the hypoconnectivity phase most strongly related to tau pathology burden ${ }^{15}$. In addition, the precuneus is one of the sites where hyperconnections and hypoconnections converge in AD and shows a hybrid pattern of high tau and high amyloid- $\beta$ pathology ${ }^{16,41}$. Taken together, one could hypothesize that elevated amyloid- $\beta$ levels, and 
perhaps associated neuroinflammation, could contribute to an increase in functional connectivity or neuronal firing between the entorhinal cortex and precuneus, increasing the vulnerability of these regions for early tau deposition in ADAD. Tau pathology may then spread from the entorhinal cortex to the precuneus along functional connections inducing dysconnectivity between these regions, and between the precuneus and other regions across the brain, ultimately resulting in behavioral and cognitive impairment. Studying the relationship between AD-related pathology and the functional organization of the brain in younger mutation carriers may provide the opportunity to test this hypothesis.

The insula also exhibited decreased segregated functional connectivity related to greater tau burden. The insula is part of the salience network (SN), which is involved in integrating internal and external stimulus. It plays a critical role in facilitating communication among diverse largescale functional brain systems (i.e., frontoparietal, ventral attention and sensorimotor networks), and serves as an integrative heteromodal association area. The insula has reciprocal connections to the entorhinal cortex and has been previously associated with decreased functional connectivity with the precuneus in preclinical $A D^{42,43}$. It has been demonstrated that the density of neurofibrillary tangles in the insula increases as accumulation of tangles in the entorhinal cortex increase, particularly when there is extensive pathology burden ${ }^{44}$. Therefore, reduced segregated functional connectivity of the insula in the presence of tau mostly in the entorhinal cortex, precuneus and inferior temporal gyrus may also be key in predicting patterns of tau propagation and possibly of neural network disintegration in ADAD.

Similar to the DMN, the SN has also been shown to undergo a phase of hyperconnectivity associated with mostly amyloid- $\beta$, followed by hypoconnectivity ${ }^{15}$. Opposite to the insula, mutation carriers exhibited greater segregated and integrated functional connectivity in other SN regions such as the anterior cingulate cortex and the striatum. The striatum is a hub that 
structurally and functionally connects many cortical structures, including DMN regions such as the medial prefrontal cortex, which also exhibited greater segregated functional connectivity, the dorsolateral prefrontal cortex and inferior parietal lobe, as well as sensorimotor networks. The striatum is highly vulnerable to early amyloid- $\beta$ accumulation in $A D^{32}$, which has been shown to predict tau pathology burden in the entorhinal cortex in ADAD, as well as lower hippocampal volume and cognitive performance in $\mathrm{MCl}^{32,33}$. Our data suggests that the striatum is hyperconnected to multiple structures within and across functional networks that are also vulnerable to early amyloid- $\beta$ accumulation, again highlighting the link between amyloid- $\beta$ and increased connectivity. The elevated strength in functional connections that we observed may also be reflecting neuroinflammatory processes known to occur early in $A D$ as a response to amyloid- $\beta$ burden and neural injury (including in the striatum) $)^{45}$, which later contribute to synaptic dysfunction and loss, as well as impaired network communication and neurodegeneration ${ }^{46-48}$. As such, it is possible that greater functional connectivity in the striatum may be an early indicator of risk for $\mathrm{MCl}$ and dementia. It is important to also highlight that there were opposite patterns of functional connectivity in regions that belong to the same network (i.e., SN), showcasing the dynamic nature of distinct hubs.

Finally, we found that greater tau burden predicted greater segregation of functional connections in sensorimotor networks, including the visual network, which have been shown to make up a robust coherent network that integrates multimodal regions ${ }^{49}$. It has been hypothesized that this network acts as an interface between external information and hubs that are involved in higher-order cognitive functions, such as those pertaining to the $\mathrm{DMN}^{49}$. In fact, prior findings have provided evidence of a link between decreased connectivity of multimodal association areas and cortical dysfunction in AD. The accumulation of amyloid- $\beta$ and tau pathology in this multimodal integrated network may thus be key for the disruption of neural communication within and across communities that lead to further pathology accumulation and 
cognitive decline. In fact, these patterns of segregated and integrated functional connectivity were significantly associated with memory performance, such that networks that strengthened or weaken as the disease progresses were related to worse memory (Figure 3), further lending support to the critical role of functional network organization in cognitive functioning.

The current study has multiple strengths. First, we did not rely on presenting symptoms, predicted risk (e.g., based on PET PiB levels or APOE genotype) or cognitive data to infer whether individuals will go on to develop dementia. Instead, we tested our hypotheses in a group of individuals who have a well-characterized clinical trajectory. Studying ADAD provides a unique opportunity to study biomarkers of $A D$ in the preclinical stage, as we can estimate how far mutation carriers are from the clinical symptom onset based on the mutation that they carry. In addition, we examined in vivo amyloid- $\beta$ and tau pathology using PET imaging, which is considered the gold standard for quantifying and mapping brain pathology in AD. Mutation carriers were also young and otherwise generally healthy, which minimizes potential confounding variables that are more common in advanced age and contribute to brain dysfunction and cognitive decline (e.g., cardiovascular disease). Finally, the very homogeneous clinical profile of mutation carriers allows us to infer how functional connections may change as the disease progresses.

While the present study has many strengths, it also has caveats that must be considered when interpreting the data. First, our sample size is relatively small compared to other studies of $A D$ and cognitive aging. However, individuals with these mutations are relatively rare and all our participants carry a single mutation (PSEN1 E280A), which makes our sample highly homogeneous compared to other cohorts, and one of the largest single mutation ADAD samples with PET imaging and fMRI. More research is also needed to examine whether our findings in ADAD generalize to preclinical late-onset AD, as well as whether APOE4 status may 
impact the results in this kindred. We are currently conducting the first longitudinal biomarker study with this cohort, which will provide greater insight into how annual change in functional connectivity relates to in vivo pathology burden and cognitive decline over time.

Collectively, these findings highlight the importance of not limiting analyses to examining group averages or specific seeds, as it can obscure the unique patterns of functional disintegration associated with disease progression. Our data also underscores that more attention must be given to the precuneus as an early marker of $A D$ and predictor of disease progression that has a key role in functional dysconnectivity. Findings enlighten our understanding of how AD-related pathology may distinctly alter the functional architecture of the brain to interfere with the integration of information across different neural systems and possibly propagate the spread of pathology, ultimately resulting in cognitive impairment and dementia.

\section{METHODS}

Study design and participants. PSEN1 E280A carriers and age- and education-matched noncarriers from the Massachusetts General Hospital COLBOS (Colombia-Boston) longitudinal biomarker study participated in this study. Participants were recruited from the Alzheimer's Prevention Initiative registry of ADAD, which currently includes more than 6,000 living members of the kindred and approximately 1,200 mutation carriers ${ }^{50}$. Exclusion criteria included a history of psychiatric disorders, illiteracy, stroke, epilepsy, traumatic brain injury, kidney failure, human immunodeficiency syndrome, or substance abuse. To be included in this study mutation carriers had to demonstrate no cognitive impairment on a standard cognitive battery, including a clinical diagnostic rating scale (CDR) score of 0, a Functional Assessment Staging Test (FAST) score of 2 or less, and a Mini-Mental State Examination (MMSE) score of 26 or greater. Demographic information is presented in Table 1. 
The study was approved by both the institutional ethics review boards of the University of Antioquia in Colombia and Massachusetts General Hospital in Boston. Participants provided signed informed consent before participating in any procedures.

All participants in this study travelled from Colombia to Boston (USA) and underwent PET imaging and MRI at the Massachusetts General Hospital. Participants and investigators were blind to the genetic status of the individuals.

Genotyping. Genomic DNA was extracted from blood using standard protocols. PSEN1 E280A characterization was done at the University of Antioquia as previously described ${ }^{51}$. Genomic DNA was amplified with the primers PSEN1-S 5' AACAGCTCAGGAGAGGAATG 3' and PSEN1-AS 5' GATGAGACAAGTNCCNTGAA 3'. We used the restriction enzyme Bsml for restriction fragment length polymorphism analysis. Each participant was classified as a PSEN1 E280A carrier or non-carrier.

Neuropsychological tasks. Cognitive testing was conducted in Spanish at the University of Antioquia by psychologists trained in neuropsychological assessment within two months of brain imaging. A Spanish version of the Consortium to Establish a Registry for Alzheimer's Disease (CERAD) word list test was used to measure episodic memory ${ }^{52}$. In the CERAD word learning list participants were required to learn 10 items over 3 trials (total recall score), a measure of encoding. Participants were then asked to recall as many words as they could from the previously learned list after a 10-minute delay (delayed recall score), which measures the ability to retrieve information. Higher scores represent better performance on the memory task. 
MRI data acquisition and preprocessing. The MRI image acquisition was performed on a Siemens 3T Tim Trio system using a 12-channel phased-array head coil. A high-resolution 3D T1-weighted magnetization prepared rapid gradient-echo (MPRAGE) sequence was used with the following parameters: $1 \mathrm{~mm}$ isotropic voxels; 160 sagittal slices; acquisition matrix size=256 $\times 256$; repetition time $(T R)=2300 \mathrm{~ms}$; echo time $(T E)=2.98 \mathrm{~ms}$; field of view $(F O V)=256$ $\mathrm{mm}$. To measure blood oxygenation level dependent (BOLD) signal a functional gradient-echo echo-planar pulse sequence was used with the following parameters: TR of 3 seconds, flip angle 858, TE $30 \mathrm{~ms}$, matrix 723 72, field of view 216 × $216 \mathrm{~mm}, 47 \times 3 \mathrm{~mm}$ axial slices, which resulted in isotropic voxels of $3 \mathrm{~mm}$ and 124 volumes lasting 6 minutes. Participants were asked to lie flat, keep eyes open, and remain awake. Bi-temporal foam pads were used to restrict head motion.

MRI data was preprocessed using FMRIB Software Library v5.0.7 (FSL) and MATLAB R2019b. The anatomical T1 preprocessing pipeline included: reorientation to right-posterior-inferior (RPI); alignment to anterior and posterior commissures; skull stripping; gray matter, white matter and cerebrospinal fluid segmentation; as well as a computation of non-linear transformation between individual skull-stripped T1 and 2mm resolution MNI152 template images. The functional MRI preprocessing pipeline included: slice timing correction; reorientation to RPI; rigid-body realignment of functional volumes within runs (6 parameters linear transformation); computation of the transformation between individual skull-stripped T1 and mean functional images using linear boundary based registration (https://fsl.fmrib.ox.ac.uk/fsl/fslwiki/FLIRT_BBR); intensity normalization; removal of confounding factors from the data using linear regression that included 12 motion-related covariates (rigid motion parameters and its derivatives), linear and quadratic terms, and five components each from the lateral ventricles and white matter. Global signal regression was not applied due to the spurious correlations this can introduce. Non-linear transformation of resting-state data to $\mathrm{MNI}$ space, concatenating the transformation from 
functional to structural and from structural to $3 \mathrm{~mm} \mathrm{MNI}$ standard space, spatial smoothing with an isotropic Gaussian kernel of $8 \mathrm{~mm}$ FWHM, and band-pass filtering $(0.01-0.08 \mathrm{~Hz})$ to reduce low-frequency drift and high-frequency noise were also performed. Head motion was quantified using realignment parameters obtained during image preprocessing, which included 3 translation and 3 rotation estimates. Scrubbing of time points with excess head motion, Jenkinson frame displacement $>0.5 \mathrm{~mm}$, were interpolated. No participant was excluded due to head motion. The distribution of the correlations across all-time series were inspected for possible noise contamination; no outliers were observed from the whole-brain connectivity distributions across participants.

\section{C Pittsburg compound B and [F18] Flortaucipir PET acquisition procedures. Each} individual PET data set was rigidly co-registered to the subject's MPRAGE MR data using SPM8 (Wellcome Department of Cognitive Neurology, Function Imaging Laboratory, London). As reported previously ${ }^{2}, 11 \mathrm{C}$ Pittsburg compound B (PiB) PET was acquired with a 8.5 to 15 $\mathrm{mCi}$ bolus injection followed immediately by a 60 -minute dynamic acquisition in 69 frames (12x15 seconds, $57 \times 60$ seconds). 11C PiB PET data were expressed as the distribution volume ratio (DVR) with cerebellar grey as reference tissue. Regional time-activity curves were used to compute regional DVRs for each region if interest (ROI) using the Logan graphical method applied to data obtained between 40 and 60 minutes after injection ${ }^{53}$. Of note, we did not have 11C PiB PET data for one participant.

[F18] Flortaucipir (FTP) images were acquired between 80 and 100 minutes after a 9.0 to 11.0 $\mathrm{mCi}$ bolus injection in 4 separate 5-minute frames. [F18] FTP specific binding was expressed in FreeSurfer (FS) ROls as the standardized uptake value ratio (SUVR) to the cerebellum, as previously reported ${ }^{54}$. The spatially transformed SUVR PET data was smoothed with a $3 \mathrm{~mm}$ Gaussian kernel to account for individual anatomical differences ${ }^{55}$. PET data were down- 
sampled to $3 \mathrm{~mm}$ in order to examine the associations with the functional connectivity data.

SUVR values were represented graphically on vertices at the pial surface.

Integration and segregation analysis. The human brain exhibits a modular organization where different subsets of voxels form communities with specialized task ${ }^{56}$. Based on this modular organization the brain connectivity can be separated in: i) segregated links connecting all the voxels belonging to the same community; and ii) integrated links connecting links of each community with the rest of the communities of the brain for higher cognitive integration.

Whole brain functional connectivity matrix was computed using Pearson $r$ correlation coefficient in the time series of each pair of voxels. A 49,314 x 49,314 association matrix was obtained for each participant and negative values were removed ${ }^{14,16}$. We created a functional brain atlas of the different resting state networks to classify each link as segregation or integration. Eight networks of interest were extracted from 100 GSP subjects ${ }^{57}$ : visual, sensory-motor, dorsal attention (lateral-visual), ventral attention (salience), limbic, frontoparietal, default mode and language. The average population map of each network was computed, and each voxel of the atlas was assigned to each network based on the maximum probability of each voxel belonging to each of the networks. If the link start and end voxels belong to the same resting state network the link was classified as segregated link otherwise the link was classified as integrated link. For each participant we computed the weighted degree of segregated and integrated links separately obtaining 2 brain maps one for each connectivity type ${ }^{58}$. The weighted degree represents the amount and strength of connections that each voxel in the brain has. Higher values in the segregated map means that that voxel have a high number and strong connecting links to other regions of the same functional network. On the other hand, high values in integrated map means that this voxel is an important hub for integration of information between brain communities. 
Association of functional properties and PET images. To find how amyloid- $\beta$ and tau burden influence functional connectivity we used partial correlations adjusting for age between individualsubject segregation and integration weighted degree maps with whole-brain PiB and FTP PET data, in mutation carriers only as non-carriers had very little variability in pathology burden. We obtained 4 different association matrices: i) relationship of tau with functional segregation; ii) relationship of tau with functional integration; iii) relationship of $\mathrm{PiB}$ with functional segregation; iv) relationship of $\mathrm{PiB}$ with functional integration. To remove non-significant associations adjusting for multiple comparisons a False Discovery Rate (FDR) threshold of $<0.05$ was applied $^{59}$. The weighted degree of the resulting links was computed to identify integrated and segregated brain hub regions associated with amyloid- $\beta$ or tau burden. A $z$ score was applied to the degree maps and values higher than 1,64 standard deviations (SD), which represents the $10 \%$ of highest values in a normal distribution, were projected to brain surface for visualization of highest impacted regions.

\section{Statistical Analyses}

Demographics and cognition. We conducted independent samples t-tests using SPSS Statistics, version 24.0 (Armonk, NY: IBM Corp.) to examine differences in age and education between PSEN1 mutation carriers and non-carriers. We also conducted a univariate analysis of variance covarying for age, a proxy of disease progression in ADAD, to examine group differences in the MMSE and CERAD word list delayed recall scores. We used Chi-squared test to examine sex and FAST differences between groups. Analyses used a family-wise significance threshold of $p<0.05$ to correct for multiple comparisons and Cohen's $d$ to calculate effect sizes. 
Neuroimaging. We conducted a general linear model to compute a two-sample t-test and compare weighted degree maps between the mutation carriers and the non-carriers covarying for age (MATLAB V9.6, The MathWorks, Inc., Natick, MA, USA). We also conducted linear regressions to examine relationships between integrated and segregated connectivity maps and age, and multiple linear regressions with age as covariate to examine relationships between integrated and segregated functional connectivity and episodic memory. We computed wholebrain correction for multiple comparisons using Monte Carlo simulation with 10,000 iterations to estimate the probability of false positive clusters with a two-tailed $p$ value $<0.05$ (3dClustSim, afni.nimh.nih.gov). 


\section{ACKNOWLEDGMENTS}

The authors thank the Colombian families for contributing their valuable time and effort, without which this study would not have been possible. We also thank Francisco Piedrahita, Alex

Navarro, Yamile Bocanegra and Claudia Ramos from Grupo de Neurociencias, Universidad de Antioquia in Medellín, Colombia, as well as Heirangi Torrico-Teave, Arabiye Artola, Jairo Martínez, and Diana Munera from the Massachusetts General Hospital in Boston, MA, for helping coordinate visits to Boston and assisting with data collection and processing. 
bioRxiv preprint doi: https://doi.org/10.1101/2021.07.03.450933; this version posted July 4, 2021. The copyright holder for this preprint (which was not certified by peer review) is the author/funder, who has granted bioRxiv a license to display the preprint in perpetuity. It is made available under aCC-BY-NC-ND 4.0 International license.

\section{AUTHOR CONTRIBUTIONS}

EGV wrote the drafts and integrated contributions from all co-authors. EGV and ID conducted data analyses. EGV, ID, YTQ and JS designed the study. All other authors participated in data collection, discussed the contents of the manuscript and contributed to editing the article. 


\section{COMPETING INTERESTS STATEMENT}

Dr. Guzmán-Vélez was supported by the National Institute on Aging (NIA) K23AG061276. Dr.

Quiroz was supported by grants from the NIH NIA (R01 AG054671], the Alzheimer's

Association, and Massachusetts General Hospital ECOR. Mr. Fox-Fuller reports NRSA support

from the NIA (1F31AG06215801A1). Dr. Vila-Castelar was supported by a grant from the

Alzheimer's Association (2019A005859). Dr. Pardilla-Delgado was supported by the NHLBI

3T32HL007901-19S1. Dr. Lopera was supported by an Anonymous Foundation, and the

Administrative Department of Science, Technology and Innovation. He is the co-principal

investigator of the Alzheimer's Prevention Initiative (API) Autosomal Dominant AD Trial, which is

supported by NIA, philanthropy, Genentech, and Roche. Dr. Sperling receives research support

for NIH grants (P01AG036694, P50AG005134, 2009-2020, and U19 AG10483), as well as from

Eli Lilly (clinical trial) and the Alzheimer's Association. She is a site principal investigator or

coinvestigator for Avid, Bristol-Myers Squibb, Pfizer, and Janssen Alzheimer Immunotherapy

clinical trials. She receives travel funding and honoraria from AC Immune, Janssen, and Roche.

She consults for Biogen, Roche, AC Immune, Eisai, Takeda, Neurocentria, and Janssen.

Spouse consults for Novartis, AC Immune and Janssen. Dr. Johnson has provided consulting

services for Novartis, Biogen, and Eli Lilly, received support from a joint NIH-Lilly-sponsored

clinical trial (A4 Study - U19AG10483), and received research support from NIH grants R01

AG027435, P50 AG00513421, AG036694, R01 AG046396, R13 AG042201174210,

U19AG10483, and U01AG024904, as well as the Alzheimer Association and Marr Foundation.

Dr. Sepulcre was supported by grants from the NIH (R01 AG061811, R01 AG061445). All other

co-authors have no conflicts or disclosures relevant to the manuscript. Dr. Schoemaker received postdoctoral fellowships from the American Heart Association (20POST35110047) and the

Fonds de recherche du Québec - Santé (254389). 


\section{REFERENCES}

1 Sperling, R. A. et al. Toward defining the preclinical stages of Alzheimer's disease: recommendations from the National Institute on Aging-Alzheimer's Association workgroups on diagnostic guidelines for Alzheimer's disease. Alzheimers Dement 7, 280-292, doi:10.1016/j.jalz.2011.03.003 (2011).

2 Quiroz, Y. T. et al. Association Between Amyloid and Tau Accumulation in Young Adults With Autosomal Dominant Alzheimer Disease. JAMA Neurology 75, 548-556 (2018).

3 Jack, C. R., Jr. et al. Longitudinal tau PET in ageing and Alzheimer's disease. Brain 141, 1517-1528, doi:10.1093/brain/awy059 (2018).

4 Braak, H. \& Braak, E. Staging of Alzheimer's disease-related neurofibrillary changes. Neurobiol Aging 16, 271-278; discussion 278-284, doi:10.1016/0197-4580(95)00021-6 (1995).

5 Grothe, M. J. et al. In vivo staging of regional amyloid deposition. Neurology 89, 20312038, doi:10.1212/wnl.0000000000004643 (2017).

6 Kaufman, S. K., Del Tredici, K., Thomas, T. L., Braak, H. \& Diamond, M. I. Tau seeding activity begins in the transentorhinal/entorhinal regions and anticipates phospho-tau pathology in Alzheimer's disease and PART. Acta Neuropathologica 136, 57-67, doi:10.1007/s00401-018-1855-6 (2018).

7 Braak, H., Thal, D. R., Ghebremedhin, E. \& Del Tredici, K. Stages of the pathologic process in Alzheimer disease: age categories from 1 to 100 years. J Neuropathol Exp Neurol 70, 960-969, doi:10.1097/NEN.0b013e318232a379 (2011).

8 Vogels, T. et al. Propagation of Tau Pathology: Integrating Insights From Postmortem and In Vivo Studies. Biological Psychiatry, doi:10.1016/j.biopsych.2019.09.019 (2019). 
9 laccarino, L. et al. Local and distant relationships between amyloid, tau and neurodegeneration in Alzheimer's Disease. Neurolmage: Clinical 17, 452-464, doi:10.1016/j.nicl.2017.09.016 (2018).

10 Franzmeier, N. et al. Functional brain architecture is associated with the rate of tau accumulation in Alzheimer's disease. Nature Communications 11, doi:10.1038/s41467019-14159-1 (2020).

11 Smith, R. et al. Resting-State Functional Connectivity Disruption as a Pathological Biomarker in Autosomal Dominant Alzheimer Disease. Brain Connect, doi:10.1089/brain.2020.0808 (2021).

12 Buckley, R. F. et al. Functional network integrity presages cognitive decline in preclinical Alzheimer disease. Neurology 89, 29-37 (2017).

13 Thomas, J. B. et al. Functional Connectivity in Autosomal Dominant and Late-Onset Alzheimer Disease. JAMA Neurology 71, 1111, doi:10.1001/jamaneurol.2014.1654 (2014).

14 Sepulcre, J. et al. Neurogenetic contributions to amyloid beta and tau spreading in the human cortex. Nature Medicine 24, 1910-1918, doi:10.1038/s41591-018-0206-4 (2018).

15 Schultz, A. P. et al. Phases of Hyperconnectivity and Hypoconnectivity in the Default Mode and Salience Networks Track with Amyloid and Tau in Clinically Normal Individuals. J Neurosci 37, 4323-4331, doi:10.1523/JNEUROSCI.3263-16.2017 (2017).

16 Sepulcre, J. et al. Tau and amyloid beta proteins distinctively associate to functional network changes in the aging brain. Alzheimers Dement 13, 1261-1269, doi:10.1016/j.jalz.2017.02.011 (2017).

17 Fredericks, C. A. et al. Early affective changes and increased connectivity in preclinical Alzheimer's disease. Alzheimer's \& Dementia: Diagnosis, Assessment \& Disease Monitoring 10, 471-479, doi:10.1016/j.dadm.2018.06.002 (2018). 
18 Buckner, R. L. et al. Cortical hubs revealed by intrinsic functional connectivity: mapping, assessment of stability, and relation to Alzheimer's disease. J Neurosci 29, 1860-1873, doi:10.1523/JNEUROSCI.5062-08.2009 (2009).

19 Berron, D., Van Westen, D., Ossenkoppele, R., Strandberg, O. \& Hansson, O. Medial temporal lobe connectivity and its associations with cognition in early Alzheimer's disease. Brain, doi:10.1093/brain/awaa068 (2020).

20 Franzmeier, N. et al. Functional connectivity associated with tau levels in ageing, Alzheimer's, and small vessel disease. Brain 142, 1093-1107, doi:10.1093/brain/awz026 (2019).

21 Harrison, T. M. et al. Tau deposition is associated with functional isolation of the hippocampus in aging. Nature Communications 10, doi:10.1038/s41467-019-12921-z (2019).

22 Vogel, J. W. et al. Spread of pathological tau proteins through communicating neurons in human Alzheimer's disease. Nature Communications 11, doi:10.1038/s41467-02015701-2 (2020).

23 Calafate, S. et al. Synaptic Contacts Enhance Cell-to-Cell Tau Pathology Propagation. Cell Reports 11, 1176-1183, doi:10.1016/j.celrep.2015.04.043 (2015).

24 Pooler, A. M., Phillips, E. C., Lau, D. H. W., Noble, W. \& Hanger, D. P. Physiological release of endogenous tau is stimulated by neuronal activity. EMBO reports 14, 389394, doi:10.1038/embor.2013.15 (2013).

25 Wu, J. W. et al. Neuronal activity enhances tau propagation and tau pathology in vivo. Nat Neurosci 19, 1085-1092, doi:10.1038/nn.4328 (2016).

26 Hanseeuw, B. J. et al. Association of Amyloid and Tau With Cognition in Preclinical Alzheimer Disease. JAMA Neurology 76, 915, doi:10.1001/jamaneurol.2019.1424 (2019). 
27 Huber, C. M., Yee, C., May, T., Dhanala, A. \& Mitchell, C. S. Cognitive decline in preclinical Alzheimer's disease: amyloid-beta versus tauopathy. Journal of Alzheimer's Disease, 1-17 (2018).

28 Sanchez, J. S. et al. Longitudinal amyloid and tau accumulation in autosomal dominant Alzheimer's disease: findings from the Colombia-Boston (COLBOS) biomarker study. Alzheimer's Research \& Therapy 13, doi:10.1186/s13195-020-00765-5 (2021).

29 Roberts, R. O. et al. Prevalence and Outcomes of Amyloid Positivity Among Persons Without Dementia in a Longitudinal, Population-Based Setting. JAMA Neurology 75, 970, doi:10.1001/jamaneurol.2018.0629 (2018).

30 Acosta-Baena, N. et al. Pre-dementia clinical stages in presenilin 1 E280A familial earlyonset Alzheimer's disease: a retrospective cohort study. Lancet Neurol 10, 213-220, doi:10.1016/S1474-4422(10)70323-9 (2011).

31 Fleisher, A. et al. P4-068: Pre-symptomatic functional brain changes in PS1 E280A mutation carriers compared with other biomarkers: Pilot data from the Alzheimer's Prevention Initiative Biomarker project. Alzheimer's \& Dementia 9, P729-P729, doi:10.1016/j.jalz.2013.05.1457 (2013).

32 Hanseeuw, B. J. et al. Striatal amyloid is associated with tauopathy and memory decline in familial Alzheimer's disease. Alzheimer's Research \& Therapy 11, doi:10.1186/s13195-019-0468-1 (2019).

33 Hanseeuw, B. J. et al. PET staging of amyloidosis using striatum. Alzheimer's \& Dementia 14, 1281-1292, doi:10.1016/j.jalz.2018.04.011 (2018).

34 Klunk, W. E. et al. Amyloid Deposition Begins in the Striatum of Presenilin-1 Mutation Carriers from Two Unrelated Pedigrees. Journal of Neuroscience 27, 6174-6184, doi:10.1523/jneurosci.0730-07.2007 (2007). 
35 Zhang, S. \& Li, C.-S. R. Functional connectivity mapping of the human precuneus by resting state fMRI. Neurolmage 59, 3548-3562, doi:10.1016/j.neuroimage.2011.11.023 (2012).

36 Utevsky, A. V., Smith, D. V. \& Huettel, S. A. Precuneus Is a Functional Core of the Default-Mode Network. The Journal of Neuroscience 34, 932-940, doi:10.1523/jneurosci.4227-13.2014 (2014).

37 Cooper, R. A. \& Ritchey, M. Cortico-hippocampal network connections support the multidimensional quality of episodic memory. eLife 8, doi:10.7554/elife.45591 (2019).

38 Cavanna, A. E. \& Trimble, M. R. The precuneus: a review of its functional anatomy and behavioural correlates. Brain 129, 564-583, doi:10.1093/brain/awl004 (2006).

39 Wu, L. et al. Dissociation between Brain Amyloid Deposition and Metabolism in Early Mild Cognitive Impairment. PLoS ONE 7, e47905, doi:10.1371/journal.pone.0047905 (2012).

40 Gusnard, D. A. \& Raichle, M. E. Searching for a baseline: Functional imaging and the resting human brain. Nature Reviews Neuroscience 2, 685-694, doi:10.1038/35094500 (2001).

$41 \mathrm{He}, \mathrm{Y}$. et al. Regional coherence changes in the early stages of Alzheimer's disease: a combined structural and resting-state functional MRI study. Neuroimage 35, 488-500, doi:10.1016/j.neuroimage.2006.11.042 (2007).

42 Xie, C. et al. Abnormal insula functional network is associated with episodic memory decline in amnestic mild cognitive impairment. Neurolmage 63, 320-327, doi:10.1016/j.neuroimage.2012.06.062 (2012).

43 Boots, E. A. et al. Cardiorespiratory fitness is associated with brain structure, cognition, and mood in a middle-aged cohort at risk for Alzheimer's disease. Brain Imaging and Behavior 9, 639-649 (2015). 
44 Bonthius, D. J., Solodkin, A. \& Van Hoesen, G. W. Pathology of the Insular Cortex in Alzheimer Disease Depends on Cortical Architecture. Journal of Neuropathology and Experimental Neurology 64, 910-922, doi:10.1097/01.jnen.0000182983.87106.d1 (2005).

45 Han, F. et al. Neuroinflammation and Myelin Status in Alzheimer's Disease, Parkinson's Disease, and Normal Aging Brains: A Small Sample Study. Parkinsons Dis 2019, 7975407, doi:10.1155/2019/7975407 (2019).

46 Taipa, R. et al. Inflammatory pathology markers (activated microglia and reactive astrocytes) in early and late onset Alzheimer disease: a post mortem study. Neuropathol Appl Neurobiol, doi:10.1111/nan.12445 (2017).

47 Heneka, M. T. Microglia take centre stage in neurodegenerative disease. Nat Rev Immunol 19, 79-80, doi:10.1038/s41577-018-0112-5 (2019).

48 Heppner, F. L., Ransohoff, R. M. \& Becher, B. Immune attack: the role of inflammation in Alzheimer disease. Nat Rev Neurosci 16, 358-372, doi:10.1038/nrn3880 (2015).

49 Sepulcre, J., Sabuncu, M. R., Yeo, T. B., Liu, H. \& Johnson, K. A. Stepwise Connectivity of the Modal Cortex Reveals the Multimodal Organization of the Human Brain. Journal of Neuroscience 32, 10649-10661, doi:10.1523/jneurosci.0759-12.2012 (2012).

50 Rios-Romenets, S. et al. The Colombian Alzheimer's Prevention Initiative (API) Registry. Alzheimer's \& Dementia 13, 602-605, doi:10.1016/j.jalz.2016.09.010 (2017).

51 Lendon, C. L. et al. E280A PS-1 mutation causes Alzheimer's disease but age of onset is not modified by ApoE alleles. Hum Mutat 10, 186-195, doi:10.1002/(SICI)10981004(1997)10:3<186::AID-HUMU2>3.0.CO;2-H (1997).

52 Aguirre-Acevedo, D. C. et al. [Validity and reliability of the CERAD-Col neuropsychological battery]. Rev Neurol 45, 655-660 (2007). 
53 Logan, J. et al. Graphical analysis of reversible radioligand binding from time-activity measurements applied to [N-11C-methyl]-(-)-cocaine PET studies in human subjects. $J$ Cereb Blood Flow Metab 10, 740-747, doi:10.1038/jcbfm.1990.127 (1990).

54 Johnson, K. A. et al. Tau positron emission tomographic imaging in aging and early Alzheimer disease. Ann Neurol 79, 110-119, doi:10.1002/ana.24546 (2016).

55 Chien, D. T. et al. Early clinical PET imaging results with the novel PHF-tau radioligand [F18]-T808. J Alzheimers Dis 38, 171-184, doi:10.3233/JAD-130098 (2014).

56 Diez, I. et al. A novel brain partition highlights the modular skeleton shared by structure and function. Scientific Reports 5, 10532, doi:10.1038/srep10532 (2015).

57 Holmes, A. J. et al. Brain Genomics Superstruct Project initial data release with structural, functional, and behavioral measures. Scientific Data 2, 150031, doi:10.1038/sdata.2015.31 (2015).

58 Bonifazi, P. et al. Structure-function multi-scale connectomics reveals a major role of the fronto-striato-thalamic circuit in brain aging. Hum Brain Mapp 39, 4663-4677, doi:10.1002/hbm.24312 (2018).

59 Benjamini, Y. \& Hochberg, Y. Controlling the False Discovery Rate: A Practical and Powerful Approach to Multiple Testing. Journal of the Royal Statistical Society. Series B 57, 289-300 (1995). 


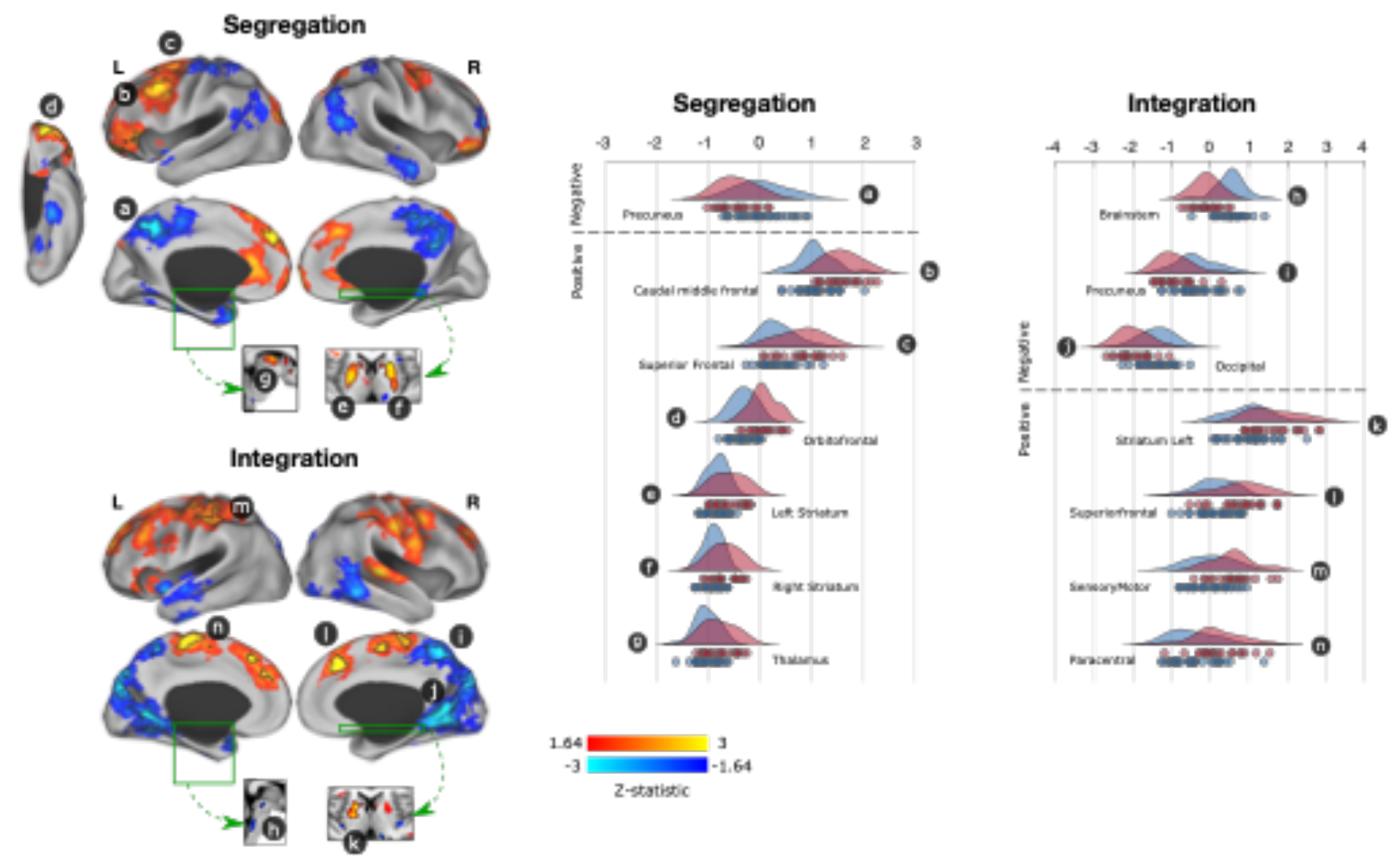

Figure 1. Differences in segregated and integrated network connectivity between cognitively-unimpaired mutation carriers and non-carriers. Less functional connectivity is illustrated in a blue color. Red-yellow color illustrate greater functional connectivity in these regions. Areas with a black border indicate the regions surviving to multiple comparisons. In Figure 1 shows differences non-carriers and mutation carriers (mutation carriers $>$ carriers). The distribution of functional connectivity and values for each individual is illustrated below the brain maps, with non-carriers being represented in blue and mutation carriers in red. 
(a) TAU Accumulation

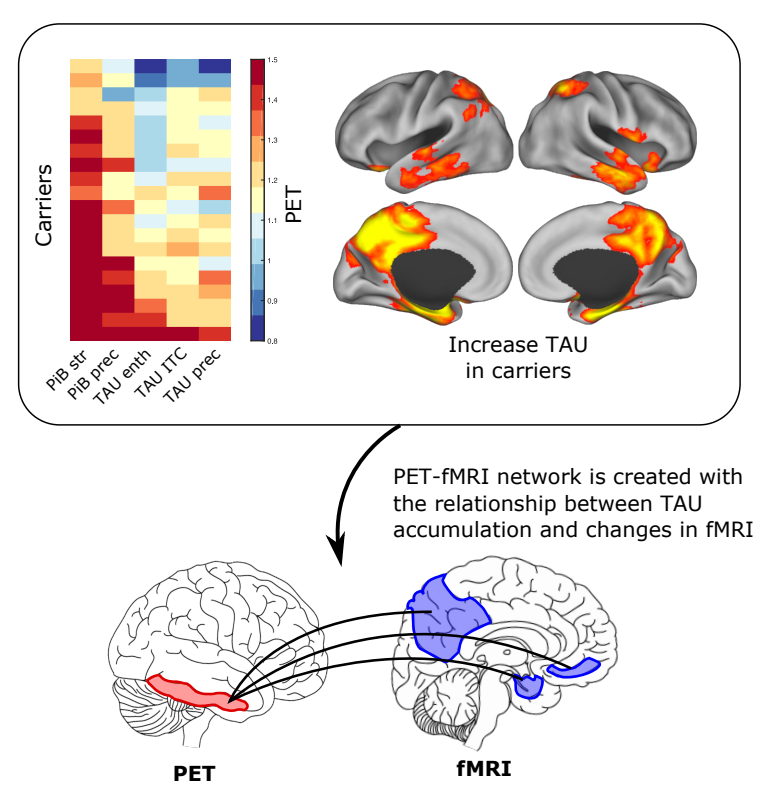

(b) TAU accumulation related fMRI changes
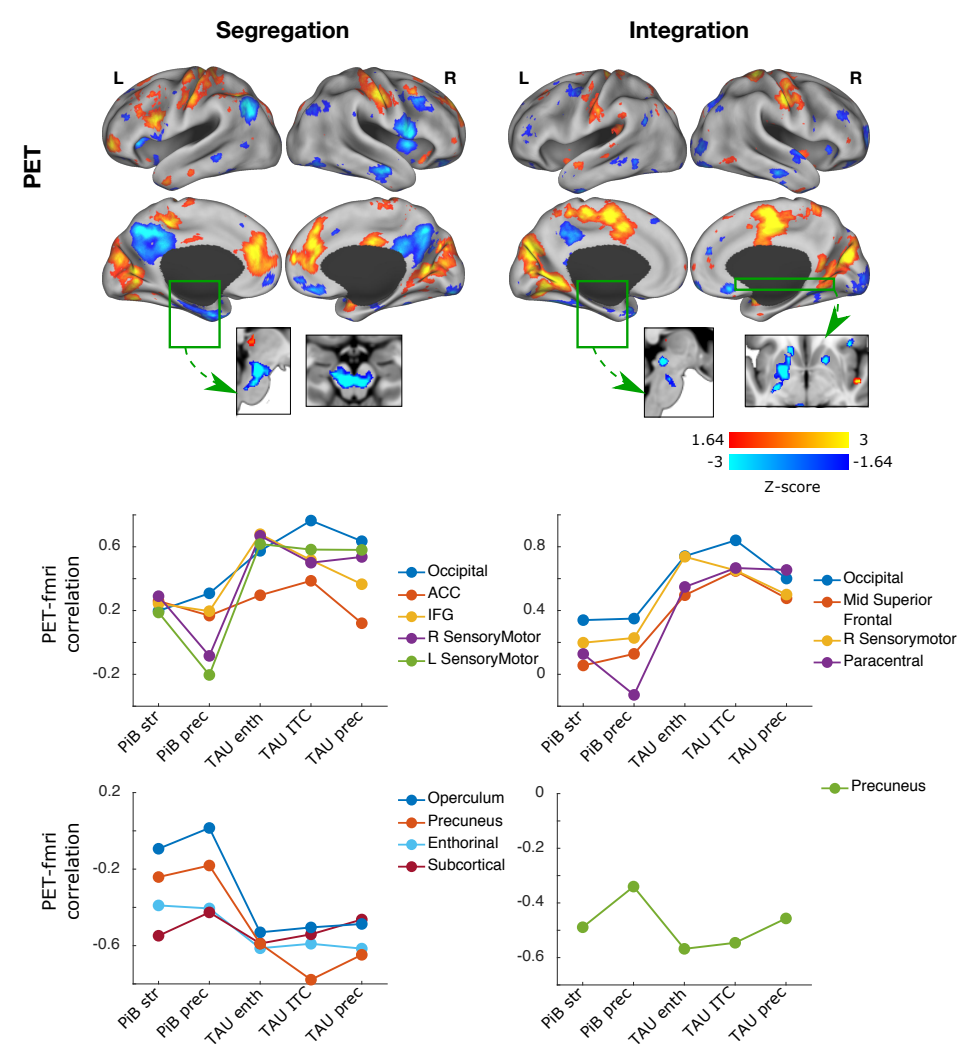

Figure 2. Relationship of segregated and integrated network connectivity, with PiB and [F18] Flortaucipir binding in mutation carriers. Figure $2 \mathrm{~A}$ illustrates $\mathrm{PiB}$ binding in the striatum and precuneus, and tau burden in the entorhinal cortex, inferior temporal gyrus and precuneus for each mutation carrier, with dark red indicating greater pathology burden and dark blue less pathology burden. It also illustrates regions where mutation carriers harbored greater tau accumulation compared to non-carriers. Figure 2B shows segregated and integrated network connectivity as predicted by tau pathology. Negative associations between variables are presented in a blue color and greater functional connectivity in red-yellow colors. It also illustrates the relationship between segregated and integrated functional connectivity in the largest clusters ( $<100$ voxels) with PiB binding in the striatum and precuneus, and tau burden in the entorhinal cortex, inferior temporal gyrus and precuneus. 

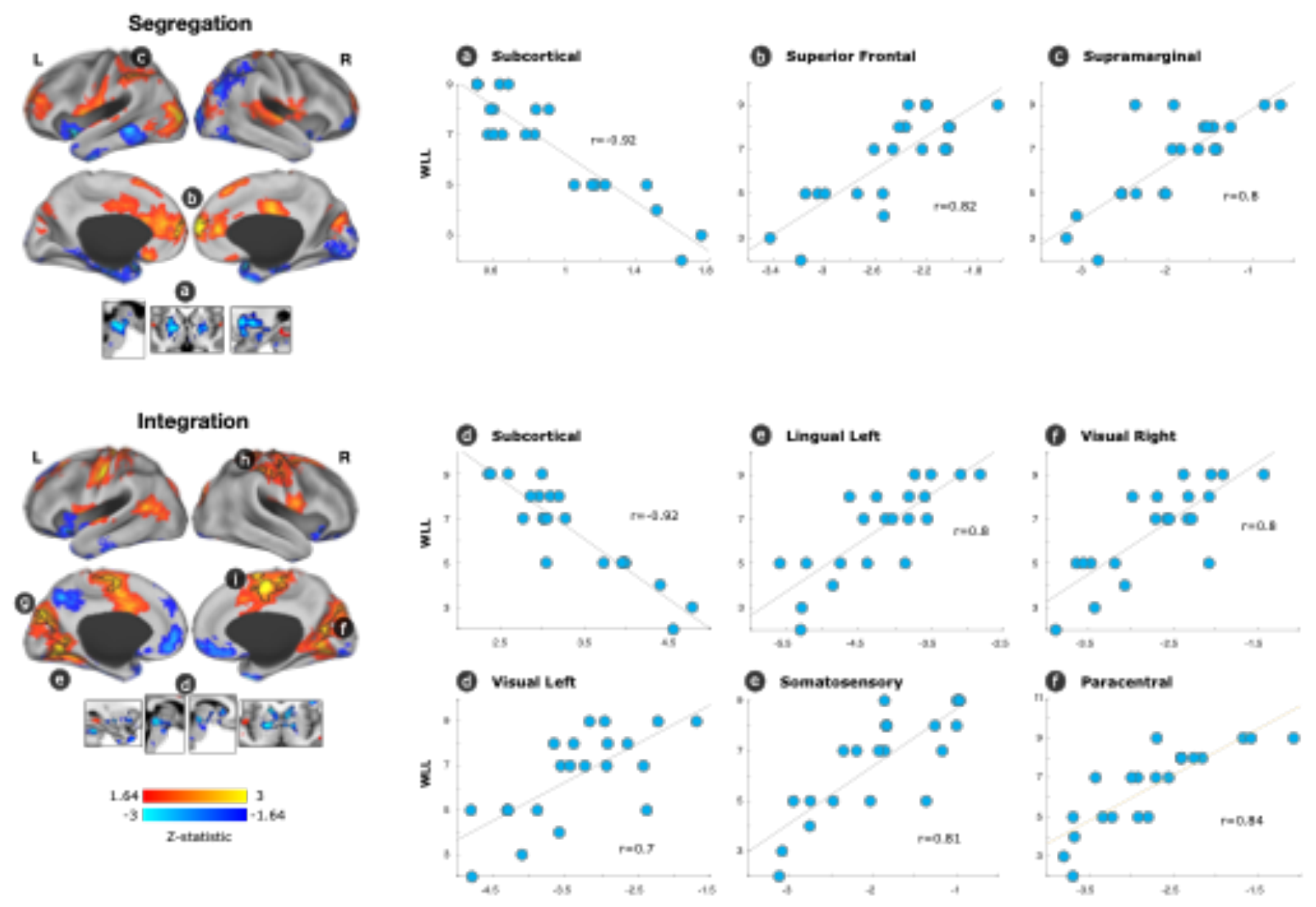

Figure 3. Association between segregated and integrated network connectivity, and word

list delayed recall in mutation carriers. Negative associations between variables are

illustrated in a blue color. Red-yellow colors illustrate increased functional connectivity in these regions. Areas with a black border indicate the regions surviving to multiple comparisons.

Scatterplots illustrate the relationship of word list delayed recall with functional connectivity in regions that survived multiple comparisons. 
Table 1. Demographic and cognitive data

$\begin{array}{cc}\text { Non-carriers } & \text { Mutation Carriers } \\ (\mathrm{n}=31) & (\mathrm{n}=21) \\ M(S D) & p\end{array}$

Demographics

$\begin{array}{lccc}\text { Age (years) } & 35.59(4.75) & 35.99(5.02) & .775 \\ \text { Education } & 11.68(4.00) & 10.19(3.84) & .185 \\ \text { Sex (\% males) } & 48.4 & 47.7 & .957\end{array}$

Cognitive tests

FAST (\% score of 1$)$

MMSE

CERAD delayed recall
93.6

$28.9(.91)$

$7.81(1.28)$
76.2

.072

$28.43(.98) \quad .085$

$6.52(2.06) \quad .007$

Note. $\mathrm{M}=$ Mean; SD = Standard Deviation; FAST = Functional Assessment Staging Test;

MMSE $=$ Mini Mental State Exam; CERAD= Consortium to Establish a Registry for Alzheimer's

Disease neuropsychological battery. Compared to non-carriers, cognitively-unimpaired mutation carriers exhibited significantly worse total and delayed recall scores. Groups did not differ between in age, education, sex, FAST or MMSE scores. 